\title{
Special aspects of explosive loosening with minimal rock displacement
}

\author{
Evgeny Shevkun ${ }^{1 *}$, Aleksander Leshchinsky ${ }^{1}$ and Andrey Plotnikov ${ }^{2}$ \\ ${ }^{1}$ Pacific State University, Chair of Transport and Technological Systems in Construction and Mining, \\ 680035, Khabarovsk city, Russia; \\ 2"AVT-Amur" Limited Liability Company, 675000, Blagoveshchensk city, Russia
}

\begin{abstract}
The dynamics of the development of an industrial mass explosion at deceleration intervals of $150 \times 200$ ms on a frame-by-frame video recording on a consumer video camera with a shooting frequency of 25 frames per second is studied. Possibilities of visual assessment of the explosion site of individual borehole charges are evaluated. The main difficulties in recording processing are caused by the difference in the intervals between frames $(40 \mathrm{~ms})$ and decelerations between charges $(50 \mathrm{~ms})$. A significant deviation of the actual deceleration intervals from the nominal value was revealed, which significantly changes the actual picture of the development of a mass explosion, including an increase in the actual development time of a mass explosion against the calculated one. The passage through the zones of the location of individual borehole charges up to 40 stress waves was established. Such multiple impacts of stress waves are expressed in a decrease in the magnitude of the interval velocities of dust and gas emissions as the number of impacts of stress waves increases. Due to the multiple effects of stress waves in the extension stage, the fracturing of the rock mass increases. This is expressed in a decrease in the dynamics, and then in the termination of the outburst of bottom hole material as the disturbance of rocks in the prefracture zone increases. There is no breakdown of rocks; the "ore-rock" contacts have retained their original position, which makes it possible to reduce the dilution of ore to a minimum.
\end{abstract}

Key words: preliminary destruction; borehole charges; deceleration intervals; ore-rock contacts.

\section{Introduction}

Explosive loosening of rocks is the main process of open pit mining, which determines the main qualitative and quantitative indicators of subsequent technological processes, primarily the excavation of ore areas with minimal dilution (mixing of waste rocks).

It is explosive loosening that determines the amount of dilution in the process of formation of the disintegration of the rock mass. Therefore, the ideal option is to loosen the rocks with minimal displacement, which allows us to maintain the primary position of the "ore-rock" contacts, refined in the process of drilling production of blocks with blast holes.

* Corresponding author: ev.shevkun@yandex.ru 


\section{Target setting}

Many years of experience in blasting operations with extended deceleration intervals at the Petropavlovsk group of companies plants showed that an increase in deceleration intervals makes it possible to reduce the disintegration of rocks to a minimum, keeping the blasted rock mass in the contours of the operational block and, thereby, to reduce the mixing of empty rocks with ores.

Let us consider the features of explosive loosening with deceleration intervals increased to $150 \times 200 \mathrm{~ms}$, using the example of a conventional massive explosion of block 12 on the mountain $+305 \mathrm{~m}$ with a volume of 32.6 thousand $\mathrm{m}^{3}$ at the Pokrovsky pit on August 21, 2016. Fig. 1 shows a scheme of initiation of 408 blast holes with a diameter of $215 \mathrm{~mm}$ and a depth of $5.5 \mathrm{~m}$, placed on a grid of $4 \times 4 \mathrm{~m}$. With a specific flow rate of $0.39 \mathrm{~kg} / \mathrm{m}^{3}$, the weight of the borehole charge was about $31 \mathrm{~kg}$, the length of stemming from drill cuttings was about $4.5 \mathrm{~m}$. The mass explosion development video was carried out with a digital camera Panasonic SDR-H100 (frequency 25 frames / s) from the overlying ledge from the side of long rows - along the green arrow at Figure 1.

The influence of multiple multidirectional effects of stress waves on the fractures of the rock mass in the vicinity of individual borehole charges was assessed by the height of the ejection of the bottom hole material from the wells and the activity of the velocity parameters of the ejection between adjacent video frames - the interval ejection velocity.

Using the graphoanalytical method [1], a sequence of blasting borehole charges was constructed according to the above diagram of the block's blasting network, and the number of stress waves passing through the area of each blast hole during the development of a massive explosion was revealed, taking into account the size of the destruction and pre-destruction zones. Within the framework of the classical concepts of the mechanism of explosive loosening of rocks, it is assumed that a crushing zone is formed around the explosion cavity, followed by a zone of radial cracks and a zone of elastic deformations. At the same time, the possibility of the formation of a transition zone of increased microcracking between the disturbed and undisturbed medium and the weakening of the rock mass under the action of multiple dynamic loads is noted [2].

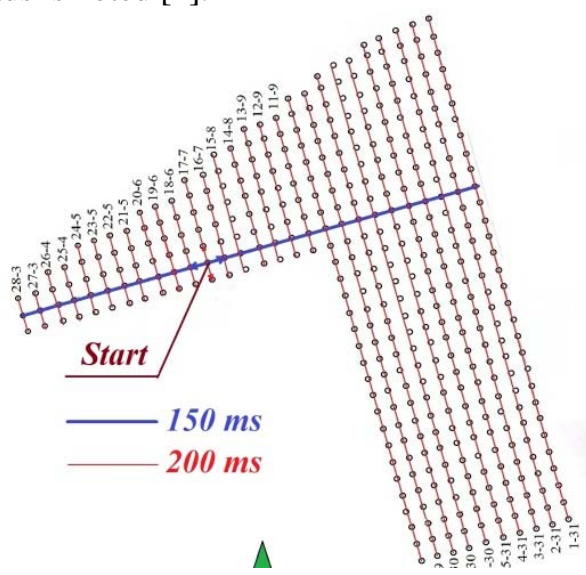

Fig. 1. Operational block network explosive scheme

The development of a massive explosion along the cut-in row with an advance due to the reduced deceleration interval does not allow tracking the emissions of the face material behind the cut-out row. Therefore, attention was paid to visualization of emissions during the development of a massive explosion along long rows (on the camera). For the analysis of the 
development of a mass explosion, of interest is the part of the block located in front of the cut row, since the influence of neighboring charges through the destruction zone affects only the second row along the cut - the third row does not affect the number of passing stress waves even in the cut row (Fig. 2), and emissions from wells behind the cut row cannot be identified. Therefore, the graphical constructions by the number of stress waves that passed through the vicinity of the blast holes were drawn from the start towards the main part of the block in three rows with the transition to the main part of the block.

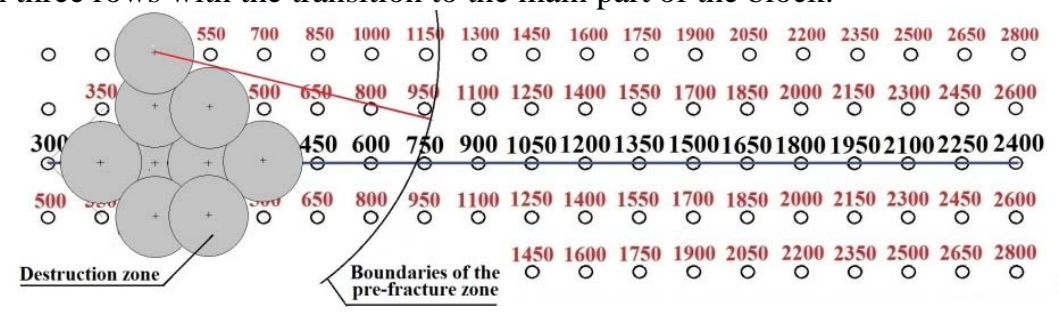

Fig. 2. Borehole charges explosion zones

Stress waves are absorbed in the destruction zone of blasted holes, which must be taken into account when constructing subsequent pre-fracture zones - they look like sectors of circles of various configurations. The rock mass in the area of wells falling into the overlap zone of the sectors of the pre-fracture zone is repeatedly exposed to stress waves. The radius of the zone of destruction of the borehole charge can reach the limiting value of 40 radii of the charge (Rch), and the zone of pre-destruction - up to (200-250) Rch [3]. We have adopted the size of the radius of the destruction zone of the borehole charge at 30 Rch $(3.2 \mathrm{~m})$, and the pre-destruction zone - 200 Rch $(21.4 \mathrm{~m})$. When blasting closely spaced borehole charges, two voltage waves from opposite directions pass through the vicinity of several borehole charges, which increases the disturbance in the pre-destruction zone of specific charges. At Figure 3, such charges are highlighted in orange.

\section{Experimental research}

Out of 224 borehole charges allocated for analysis, outbursts of bottom hole material were observed only from 78 wells. It was possible to confidently measure and process the parameters of emissions for 62 well charges (79\%), which can be considered a quite acceptable result. The number of measurement points for specific emissions varied from 4 to 18 and was associated either with the escape of the "plume" of the emission outside the frame, or with the release of the interval velocity to zero values.

A number of factors lead to the probabilistic nature of the location of the borehole charges with the measured parameters of the ejection of the bottom hole material on the block diagram. The position of a specific borehole charge inside the frame can be estimated precisely at the moment of the appearance of a dust and gas outburst; it is necessary to take into account the speed of video recording and the deviation of the response time of the detonator retarders from the nominal value. The frame-by-frame scan of the explosion videogram in $40 \mathrm{~ms}$ does not coincide with the interval of deceleration of the surface network, which is a multiple of $50 \mathrm{~ms}$, and outliers with a clearly increased height were noted in several frames (by the sum of two frames). Therefore, it was not the abnormally high speed of the first frame that was taken into account for the analysis of the dynamics of the ejection of the material from the wells, but the next peak of the speed. So, for well 7 in Figure 4, the velocity peak taken into account was $108 \mathrm{~m} / \mathrm{s}$, and not $236 \mathrm{~m} / \mathrm{s}$. All three wells are in the zone through which 9 stress waves have passed, and the diagrams for wells 8 and 9 are very similar for the first 5 
points; if the first blowout of well 7 is halved, then all three diagrams will be identical, which confirms the validity of the adopted approach to the choice of the maximum ejection rate for the calculations.

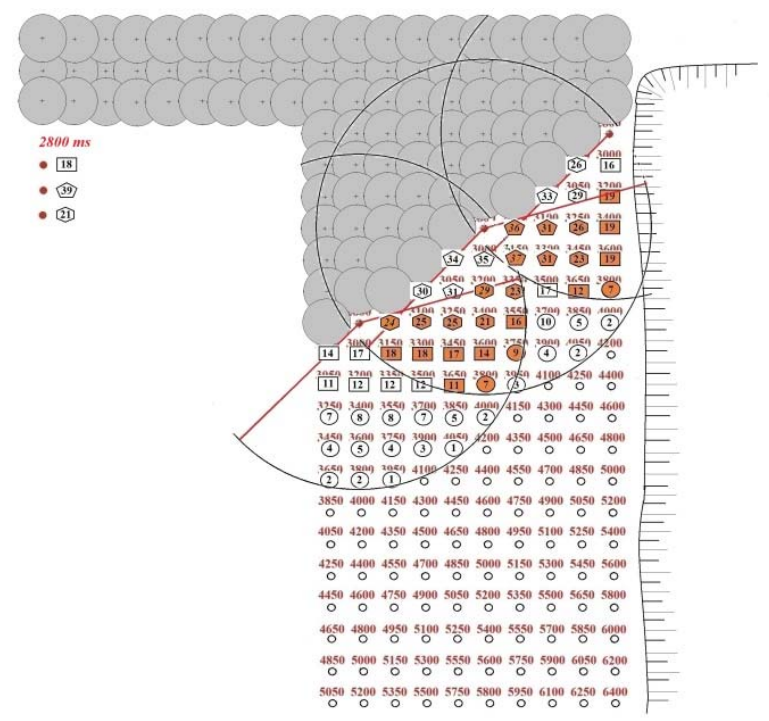

Fig. 3. The number of stress waves passing through the vicinity of the borehole charges by $2800 \mathrm{~ms}$

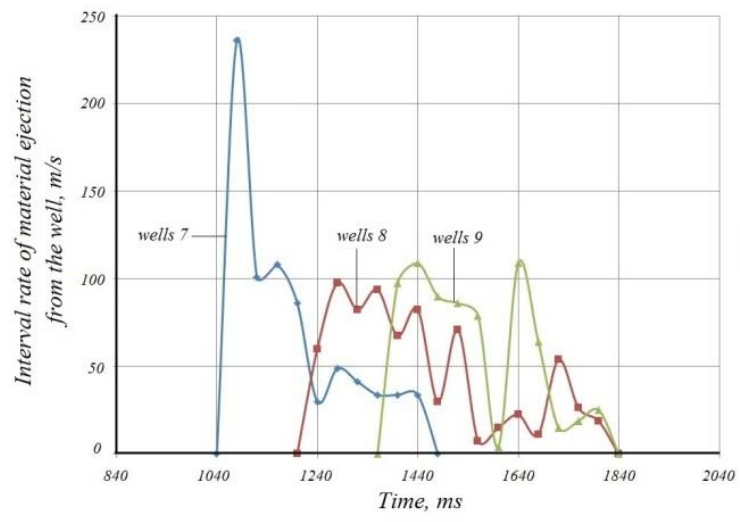

Fig. 4. Dynamics of ejection of bottom-hole material from wells 7-9.

Deviation of deceleration intervals from the nominal is the second significant factor that makes it difficult to visualize the location of a blowout from specific wells. Figure 2 shows the beginning of the switching circuit of the explosive network at nominal: $150 \mathrm{~ms}$ in the cut row and $200 \mathrm{~ms}$ in the perpendicular direction, and from Figure 4 it can be seen that the circuit operates at actually $160 \mathrm{~ms}$ in the cut. The manufacturer of the Rionel system allows deviations for surface retarders of 150 and $200 \mathrm{~ms} \pm 12.5 \mathrm{~ms}$, and $750 \mathrm{~ms}$ downhole retarders can be triggered from 725 to $800 \mathrm{~ms}$. Comparison of deceleration intervals according to actually measured borehole charges with the calculated ones showed that this batch of surface retarders works closer to the interval of 160x210 ms (Fig. 5). 


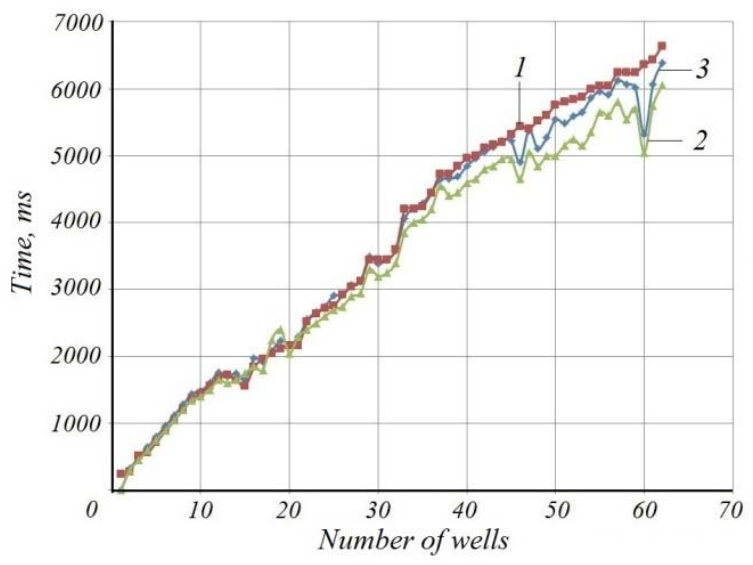

Fig. 5. Deceleration time of the of the measured wells graphs: actual (1), calculated at nominal $150 \times$ 200 (2) and calculated at maximum deviation $160 \times 210$ (3)

Until $2000 \mathrm{~ms}$, all three graphs coincide in a way, then the nominal value graph lags behind, and after $5000 \mathrm{~ms}$ the maximum deviation graph also lags behind. The sharp deviations in the response time of wells 46 and 60 can be explained by deviations from the rating and downhole retarders. At [4], it is stated that the value of the standard deviation of the retarders from the nominal value accumulates as the number of series-connected retarders increases, through which the initiating pulse approaches a given charge. This means that the more retarders connected in series are involved in the blasting scheme, the less they play their role as regulators of short-delay blasting, ensuring the quality of crushing of the rock mass. This conclusion is also confirmed with our observations: at Figure 5, this accumulation can be traced quite confidently: with the estimated response time of the last downhole charge of the block according to the scheme $160 \times 210 \mathrm{~ms} 6760 \mathrm{~ms}$, an outburst was recorded (but not measured for objective reasons) on a frame of $7160 \mathrm{~ms}$.

Figure 6 shows the layout of the wells on the block with the measured parameters of the outburst of drilling material.

Comparison of the dynamics of dust and gas emissions for different groups of wells showed a regular decrease in the interval velocities of dust and gas emissions: in Figure 7, and with the disturbance of the vicinity of wells 10-21 from the passage of 12-14 stress waves (in Figure 6 highlighted in orange) the speeds vary from 225 to $100 \mathrm{~m} / \mathrm{s}$; in Figure 7, b, in the vicinity of wells 24-30 (in Figure 6, green color), after the passage of 21-39 stress waves, the maximum interval velocities decreased to $100-50 \mathrm{~m} / \mathrm{s}$.

Upon reaching the same disturbance of rocks from the passage of stress waves, the dynamics of the ejection rates does not change along the block length: at Figure 8, after the passage of 34 stress waves through wells 31-62 (blue in Figure 6), the maximum ejection velocities fluctuate in the interval of $100-68 \mathrm{~m} / \mathrm{s}$ for 14 rows of wells (56 m) along the block. 


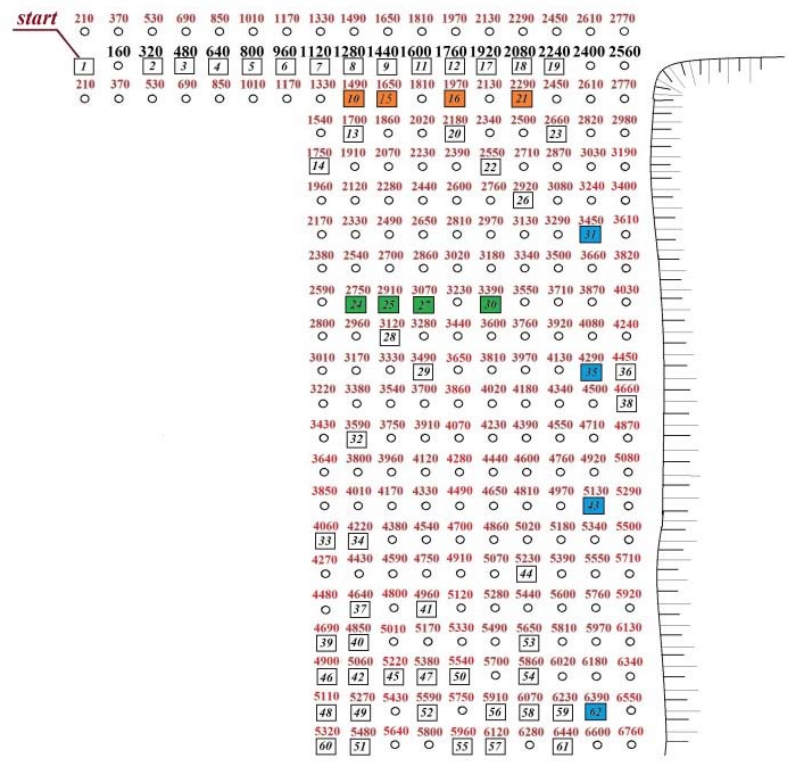

Fig. 6. Position of wells with measured emission parameters on the block

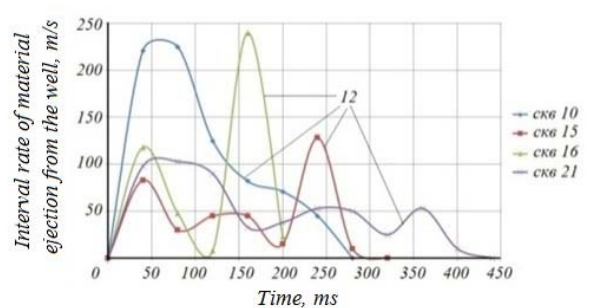

$a$

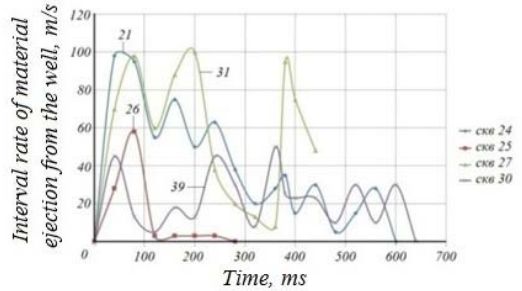

$b$

Fig. 7. Dust and gas emissions from wells across the block width dynamics

\section{Results discussion}

15 years of our experience in conducting experimental mass explosions allows us to consider video filming one of the simplest and most informative research methods. At [5], the use of interval (between each frame) speed is substantiated as the most informative indicator of the dynamics of changes in speed processes, primarily dust and gas emissions from blast holes.

The action of the stress wave was taken into account in the zone of the pre-destruction sector, limited by the tangents from the charge to the destruction zone of the previous exploded charges [6]. The impact of each impulse causes a certain number of disturbances, both as a result of the development of disturbances existing in the rock under the influence of a direct compression wave, and the formation of new ones in places of concentration of stresses, dislocations, weakened strength, etc. when exposed to a tensile wave that follows a compression wave after a certain period of time [7]. Such an impact allows a serious weakening of the massif in the pre-fracture zone. 


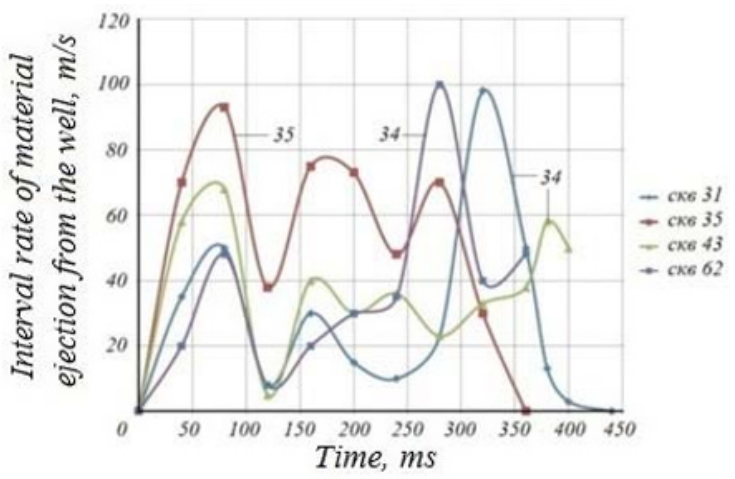

Fig. 8. Dust and gas emissions from wells across the block length dynamics

In general, this is expressed by the excess of the disintegration surface above the step surface only in the launch area by 1.5-1.8 $\mathrm{m}$ and a slight (0.3-0.4 $\mathrm{m}$ ) excess on the rest of the disintegration surface, as well as in the complete preservation of the structure of a primary massif in the disintegration of the rock mass, remaining in the block outline.

\section{Conclusions}

The analysis of the industrial explosion video confirmed the opinion expressed by the researchers that the waveguide systems of non-electric initiation with pyrotechnic retarders, due to significant deviations of their nominal value already at the initial stage of the explosion development, transfer the functions of a short-time-slow blasting regulator, which ensures the quality of crushing of the rock mass, to the function of increasing the total duration of the explosion.

Nevertheless, the development of a mass explosion in the mode "one well - one stage of deceleration" with deceleration intervals of $150 \times 200$ ms creates a minimum displacement of the rock mass in the disintegration of broken rock both vertically and horizontally - the surface of the disintegration is slightly swollen, there is no displacement outside the block. This allows us to preserve the primary structure of the rock mass in the disintegration and, thereby, provide the possibility of selective extraction of minerals in the face.

\section{References}

1. E.B. Shevkun, A.V. Leshchinsky, E.A. Shishkin, Yu.A. Lysak, Blasting work. 127/78, 3347 (2018)

2. M.A. Sadovsky, V.V. Adushkin, A.A. Spivak, Mechanical effect of an explosion. 323-329 (1994)

3. A. V. Yurovskikh, Author's summary ... PhD in tech. Sciences (2003)

4. A.A. Sysoev, Universities papers. Journals of Mining, 4, 60-67 (2016)

5. E.B. Shevkun, A.V. Leshchinsky, Yu. Gorbulya, Bulletin of Togu, 2(37), 137-146 (2015)

6. E.B. Shevkun, A.V. Leshchinsky, Yu. A. Lysak, A. Yu. Plotnikov, Mining information and analytical bulletin, 4, 272-282 (2017)

7. M.A. Novikova Author's summary. ... PhD in tech. Sciences (1984) 